\title{
Drying intensification by vibration: fundamental study of liquid water inside a pore
}

\author{
Chen, W. ${ }^{\text {a }}$ Colin, J. $^{a^{*}}$; Casalinho, J. ; Ben Amara, M.E.A. ; Stambouli, M. ${ }^{\text {a }}$ Perré, P.a \\ a LGPM. CentraleSupélec. Université Paris-Saclay, Gif-sur-Yvette, France \\ ${ }^{b}$ LESTE. École Nationale d’Ingénieurs de Monastir. Université de Monastir, Tunisie. \\ *E-mail of the corresponding author: julien.colin@centralesupelec.fr
}

\begin{abstract}
Vibration is a promising way to intensify the drying process through heatingup due to viscous dissipation, activation of internal liquid transfer and increase of external transfer. To better assess the possible contribution of these effects, we choose a multiscale approach.

This paper is focused on the pore scale, simulated by a capillary tube partially filled with water subjected to sinusoidal vibrations. We studied the displacement of water inside this tube through image analysis. This configuration mimics the moisture transfer inside the pores of a porous media during drying.

The experimental device developed in this study is applicable to a wide range of configurations, such as symmetrical or asymmetrical vibrations.
\end{abstract}

Keywords: drying intensification, acoustic vibration, pore scale, symmetrical and asymmetrical signals, image processing. 


\section{Introduction}

Vibration is a promising way for the intensification of drying [1], especially for porous media. The present work is focused on the study of the effect of vibration to assist moisture migration inside these materials. Several phenomena may be involved [2,3] such as internal heat generation by viscous dissipation, intensification of transfer at the exchange surfaces, activation of vapor diffusion by oscillating bulk velocity, change of wetting angle, etc. In this paper, we focus on the liquid water behavior inside a unique pore during vibration.

\section{Materials and Methods}

The experimental set consists of a capillary glass tube partially filled with water, a vibration chain and an optical bench controlled by a PC.

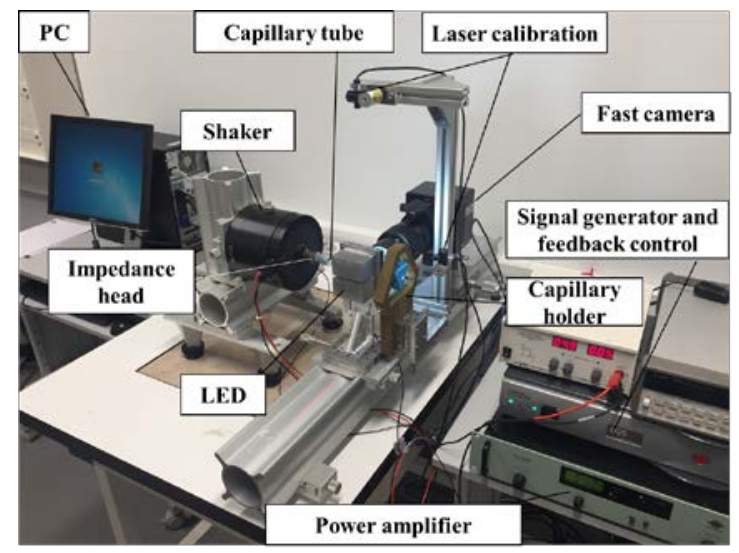

Fig. 1 A general view of the experimental device.

\subsection{Capillary tube and water index}

Capillary tubes - inner diameter $(I D)=0.6$ or $2.2 \mathrm{~mm}$, length $=15 \mathrm{~cm}$, carefully cleaned with Mucasol solution ( $3 \%$, ROTH) - are used for simulating a unique pore. Considering the difficulty of tracking the position of a smooth tube, a notch is machined on the top external wall as a mark to observe its movement. An index of distilled water is placed in this tube. In the present work, a constant index length was used, in the range 10.1 to $10.2 \mathrm{~mm}$.

\subsection{Vibration chain}

A Brüel \& Kjær vibration chain (Fig. 1) vibrates the capillary tube horizontally. A power supply (type 2718) amplifies the signal from the generator (LDS LASER ${ }_{U S B}, 64$ bits) to the vibration exciter (type 4809, maximum $8 \mathrm{~mm}$ peak-to-peak displacement, from $10 \mathrm{~Hz}$ to 20 $\mathrm{kHz}$ ). An impedance head (type 8001, with a piezoelectric accelerometer and force gauge sharing the same housing, operating frequency range up to $10 \mathrm{kHz}$ ) - fastened between the 
exciter and the tube, and connected to the signal generator - allows a feedback control. At the other end of the tube, a 3D-printed holder reduces the vertical oscillations due to the gravity and possible resonances. Series of tests were performed with sinusoidal vibrations:

$$
x_{\text {cap }}(t)=A \sin (\omega t)
$$

where $A$ is the amplitude, $\omega$ the angular frequency and $f$ the frequency $(\omega=2 \pi f)$.

\subsection{Image acquisition system}

The tube and liquid movement are monitored thanks to an optical bench, comprising a highspeed camera (FASTCAM Mini AX100, by Photron: up to $4000 \mathrm{fps}$ at full resolution of $1024 \times 1024$ with a grey level coded on 12 bits), controlled by a PC, and a backside illumination. The perpendicularity of the tube and the optical bench axis is calibrated using two line lasers.

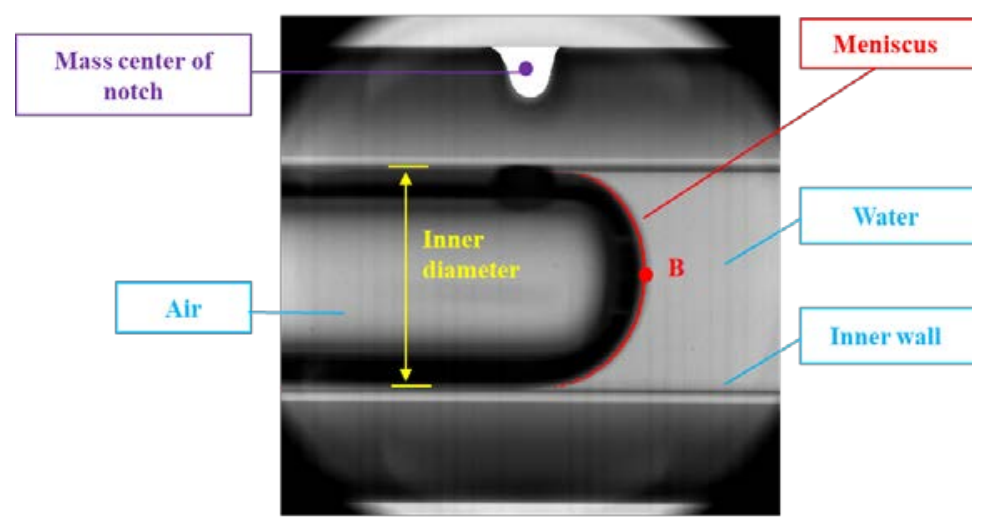

Fig. 2 Water index meniscus inside a capillary tube.

\subsection{Image processing}

The behavior of the water index is studied by image processing through the displacement of the meniscus (Fig. 2), i.e. the interface between water and air. Obtained images are analyzed using a custom Matlab program. The core of the program consists in the recognition of the inner wall, the meniscus and the notch. The boundaries between phases are characterized by a transition from light to dark. Point B is the intersection of the tube axis and the meniscus. The coordinate of the mass center of the notch (white area in Fig. 2) is used to follow the position of the capillary tube.

\section{Raw data}

For each test, we register 50 pictures per period over a total of 7 periods. Fig. 3 presents images obtained for $I D=2.2 \mathrm{~mm}, f=30 \mathrm{~Hz}$ and $A=2 \mathrm{~mm}$. Red arrow depicts the tube direction and the blue one shows the water direction compared with the previous position. 
For this test, we notice that the menisci are not perfectly symmetrical to the horizontal plane: gravity is not negligible anymore for the $2.2 \mathrm{~mm}$-inner diameter tube.

$t_{0}$

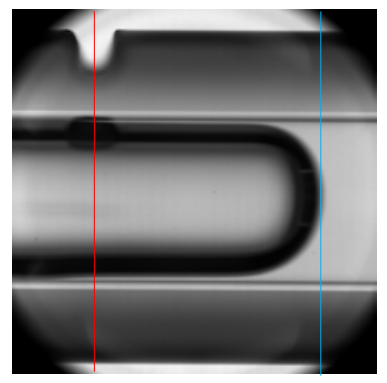

$t_{0}+6.67 \mathrm{~ms}$

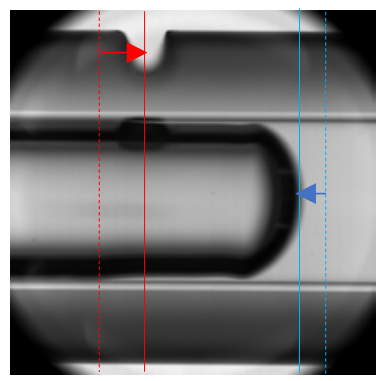

$t_{0}+13.33 \mathrm{~ms}$

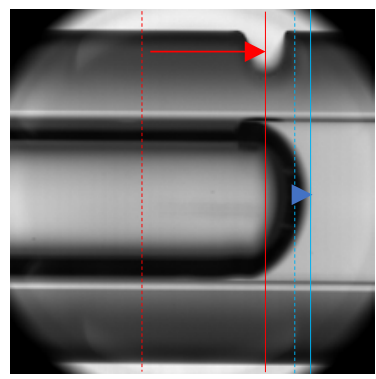

Fig. 3 Displacement of the water index during half period (ID $=2.2 \mathrm{~mm}, f=30 \mathrm{~Hz}, A=2 \mathrm{~mm}$ )

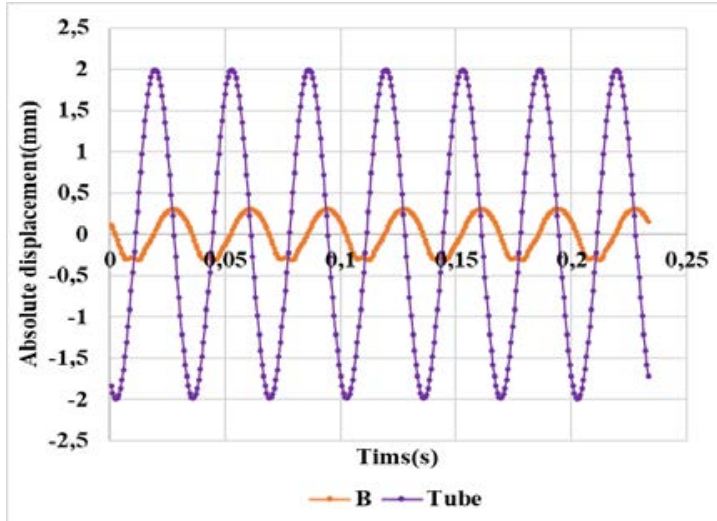

Fig. 4 Absolute displacements of water and tube vs. time $(I D=2.2 \mathrm{~mm}, f=30 \mathrm{~Hz}, A=2 \mathrm{~mm})$

We can also notice that the absolute displacement amplitude of the water index is lower than the one of the capillary tube. At this high frequency, inertia dominates the forces acting on the system (viscous and capillary forces). This observation is confirmed by all positions determined by image processing. In this case, as the absolute movement of water (Fig. 4) is much smaller than the tube movement, the relative movement of water inside the tube has almost the same amplitude as the imposed movement. For these parameters, the phase change of water movement is about $\pi / 2$.

\section{Results}

In order to study a wide range of contrasted situations, the values of three parameters were systematically tested: inner diameter (0.6 and $2.2 \mathrm{~mm})$, frequency $(10,20$ and $30 \mathrm{~Hz})$ and amplitude (0.5, 1 , and $2 \mathrm{~mm})$. Thus, we obtained a dataset of 18 experiments. The images

$21^{\text {ST }}$ INTERNATIONAL DRYING SYMPOSIUM EDITORIAL UNIVERSITAT POLITÈCNICA DE VALÈNCIA 
analysis for all tests depicts a high diversity of water index behavior, especially for menisci shape and displacement amplitude. To explain this variability, it is mandatory to quantify the relative importance of three parameters: surface tension, viscosity and inertia. For this purpose, we introduce two dimensionless numbers:

\subsection{The capillary effect}

$N_{c}$ is the ratio of capillary force $F_{c}$ over inertia $F_{i}$ :

$$
\begin{array}{ll} 
& F_{c}=2 \pi R \sigma \\
F_{i} & =m a=\pi R^{2} l \rho A \omega^{2} \\
\text { hence } \quad N_{c} & =\frac{2 \sigma}{R l \rho A \omega^{2}}
\end{array}
$$

where $R$ is inner radius of capillary, $\sigma$ the surface tension, $l$ the length of the index, and $\rho$ the water density.

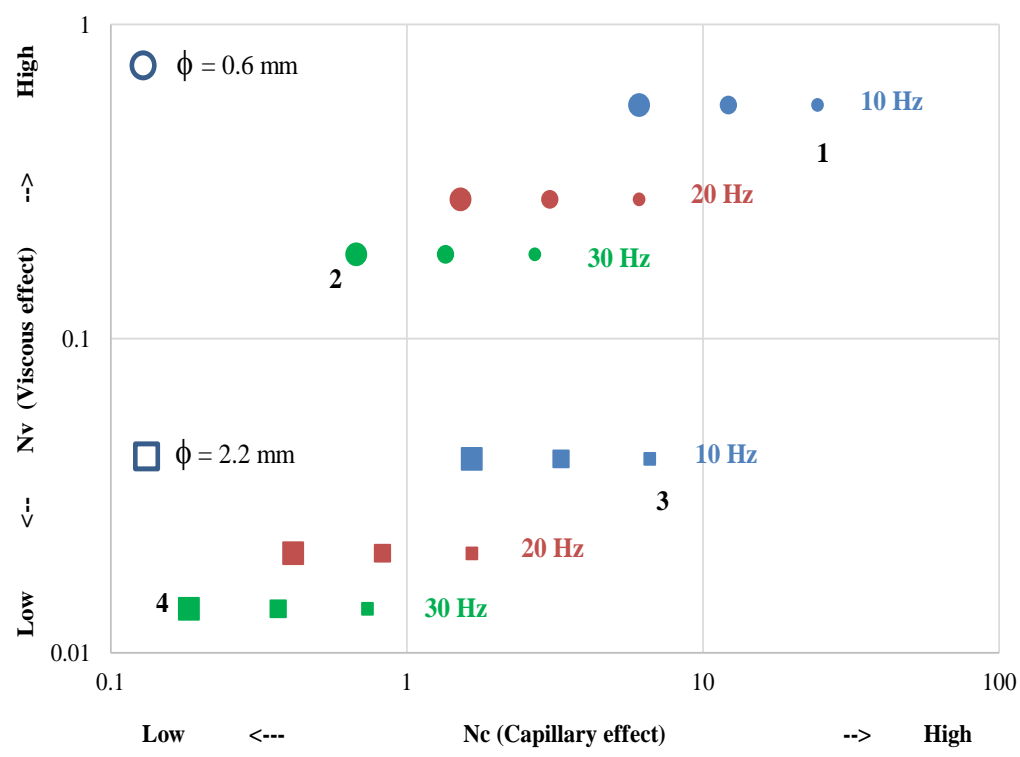

Fig. 5 Representation of all tests performed in the present study according to their respective effects of capillary forces and viscosity; One marker size per signal amplitude, (small $=0.5 \mathrm{~mm}$, medium = $1 \mathrm{~mm}$ and large $=2 \mathrm{~mm}$ )

\subsection{The viscous effect}

$N_{v}$ is the ratio of the characteristic time of the signal (half-period) over the characteristic diffusion time: 


$$
N_{v}=\frac{\mu}{2 f \rho R^{2}}=\frac{\delta^{2}}{R^{2}}
$$

where $\mu$ is the dynamic viscosity of water and $\delta$ the thickness of the Stokes boundary layer.

All tests performed in this study can therefore be represented by one marker in the plane defined by the values of $N_{c}$ and $N_{v}$ (Fig. 5). From all these tests, four contrasted situations are selected for an in-depth study.
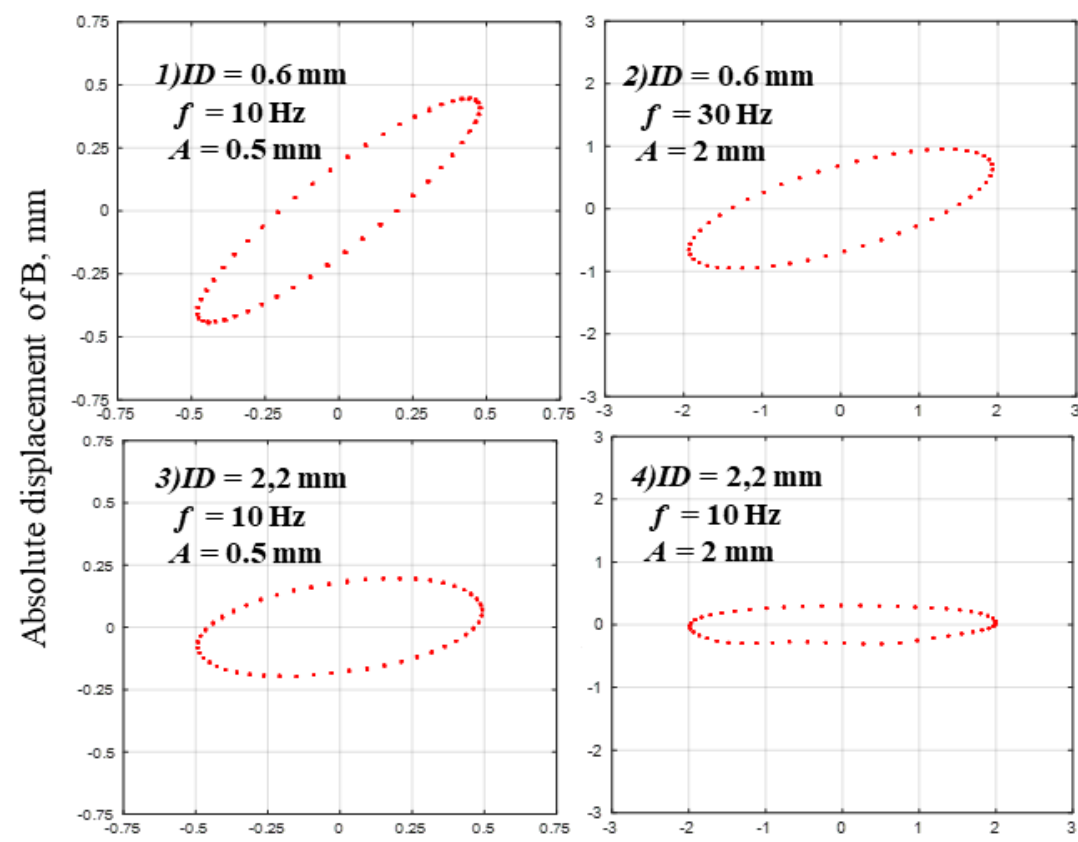

Absolute displacement of tube, $\mathrm{mm}$

Fig. 6 Lissajous curves (absolute displacement of point B plotted versus tube displacement) for the four configurations selected for the in-depth analysis (points in Fig. 5)

\subsection{Focus on contrasted situations}

In order to provide a more in-depth analysis of the relative effects of the physical phenomena (viscosity, surface tension, inertia), Lissajous curves were plotted for each selected test (Fig. 6). These curves consist in plotting the absolute displacements of the water index (point B) as a function the tube displacement. On these closed curves, we can easily observe the ratio between the tube and water amplitude (respectively width and height of the ellipse according $x$ and $y$ ) and the phase change between the signal (twice the angle between the first bisector and the main axis of the ellipse) 
For test 1 , the capillary effect dominates inertia and the viscous effect is not negligible (see the position of point 1 in Fig. 5). As a result, the water index flows the tube movement with a slight hysteresis. This explains why the curve is long and narrow along the direction $45^{\circ}$ : almost same amplitudes and same phase. For the same tube diameter $(0.6 \mathrm{~mm})$ but a larger frequency and a larger amplitude (test 2), $N_{c}$ is smaller than the unit. Consequently, the water index is not able anymore to follow the tube movement: the water index amplitude is ca. half the signal amplitude and the phase change increases. For test 3, as seen in Fig. 5, the capillary effects are quite important, but the viscous effects are now negligible, due to the larger tube diameter. Finally, test 4 lies at the very bottom-left of Fig. 5, which means very weak viscous and capillary forces. Accordingly, the water index is almost at rest in spite of the tube movement.

\section{Conclusions and perspectives}

In this work, we highlighted on the effect of vibrations on the movement of a water index in a capillary tube.

Then, various parameters were tested: tube diameter, frequency and amplitude of the tube movement. In order to quantify the effects of the parameters, two dimensionless numbers were introduced to see the effect of viscous and capillary forces compared to the index inertia. All data collected in four contrasted selected tests are perfectly explained by these dimensionless numbers.

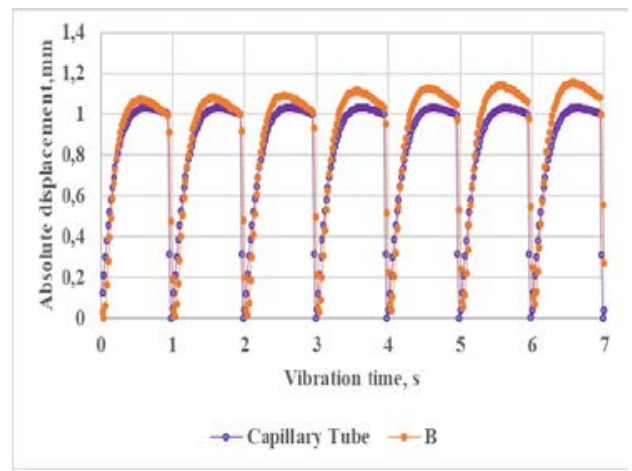

Fig. 7 Absolute displacements of $B$ and tube $(I D=2.2 \mathrm{~mm}, f=1 \mathrm{~Hz}, A=1.03 \mathrm{~mm})$

Finally, by using an asymmetrical signal, we prove that the index movement can be forced in a chosen direction. In this sense, Fig. 7 depicts a preliminary, but promising test. Using a saw-tooth wave at low frequency, we obtained an obvious global movement of the index to the right along the successive periods. This trend was inverted when changing the phase of the signal, which proves that the water movement is really due to the asymmetric signal. 
Additional tests as well as modeling approaches are in progress in our team to further investigate the effect of vibrations on liquid movement, and the numerical validation of some experimental results has also been done.

\section{Nomenclature}

\begin{tabular}{lll|lll}
$a$ & acceleration & $\mathrm{ms}^{-2}$ & $m$ & mass & $\mathrm{kg}$ \\
$A$ & sine & $\mathrm{m}$ & $N_{c}$ & capillary effect & \\
$f$ & frequency & $\mathrm{Hz}$ & $N_{v}$ & viscous effect & \\
$F$ & force & $\mathrm{N}$ & $R$ & inner radius & $\mathrm{m}$ \\
$I D$ & inner diameter & $\mathrm{m}$ & $t$ & time & $\mathrm{s}$ \\
$l$ & length & $\mathrm{m}$ & $x$ & coordinate along the & $\mathrm{mm}$
\end{tabular}

Greek letters

$\delta \quad$ Stokes boundary layer $\mathrm{mm}$

$\mu \quad$ dynamic viscosity Paxs

$\rho$ density

$\mathrm{kgm}^{-3}$

$\sigma \quad$ surface tension

$\mathrm{Nm}^{-1}$

$\omega \quad$ angular frequency

Subscripts

c capillary

cap capillary tube

i inertia

\section{References}

[1] Kroehnke J.; Radziejewska-Kubzdela E.; Musielak G., Stasiak M.; Bieganska-Marecik R. Ultrasonic-assisted and microwave-assisted convective drying of carrot-drying kinetics and quality analysis. In Proceedings of 5th European Drying Conference, Budapest, Hungray, October 21-23, 2015, 171-178.

[2] Lebovka, N.; Eugene V.; Farid C. Enhancing extraction processes in the food industry. CRC Press, 2011.

[3] Suzuki K.; Hosaka H.; Yamazaki R. Drying characteristics of particles in a constant drying rate period in vibro-fluidized bed. Journal of Chemical Engineering of Japan, 1980, 13(2): 117-122. 